\title{
CD4+/CD56+ Hematodermic Neoplasm Presenting in the Skin: A Tunisian Case Report and Current Review of the Literature
}

\author{
Yosra Ben Youssef ${ }^{1}$, Nessrine Ben Sayed ${ }^{1}$, Kmira Zahra ${ }^{1}$, Abir Gmidène ${ }^{2}$, Naouel Ben Salah ${ }^{3}$, \\ Atef Ben Abdelkader ${ }^{4}$, Nejia Brahem ${ }^{5}$, Hlima Sennana ${ }^{2}$, Colanda Belajouza ${ }^{6}$, Abderrahim Khelif ${ }^{1}$ \\ ${ }^{1}$ Department of Clinical Hematology, Farhat Hached University Hospital, Sousse, Tunisia; ${ }^{2}$ Department of Cytogenetics, Farhat \\ Hached University Hospital, Sousse, Tunisia; ${ }^{3}$ Department of Cytology, Aziza Othmana University Hospital, Tunis, Tunisia; ${ }^{4}$ De- \\ partment of Anatomopathology, Farhat Hached University Hospital, Sousse, Tunisia; ${ }^{5}$ Department of Cytology, Farhat Hached Uni- \\ versity Hospital, Sousse, Tunisia; ${ }^{6}$ Department of Dermatology, Farhat Hached University Hospital, Sousse, Tunisia. \\ Email: yosra.benyoussef@laposte.net, nessrinebensayed@yahoo.fr,kmirazahra@yahoo.fr, gmidene_abir@yahoo.fr, \\ abkheli@gmail.com
}

Received October $10^{\text {th }}, 2012$; revised November $15^{\text {th }}, 2012$; accepted November $25^{\text {th }}, 2012$

\begin{abstract}
The CD4+/CD56+ hematodermic neoplasm is a rare aggressive systemic neoplasm for which effective therapies have not yet been established, it is clinically characterized by cutaneous involvement with spread to bone marrow, blood and poor prognosis with current chemotherapy regimens. Our objective is to report diagnosis and treatment difficulties of CD4+/CD56+ hematodermic neoplasm. We describe here a Tunisian man who presented with subcutaneous ulcerated lesion localized in the right leg and multiples generalized nodules. Skin biopsy showed an atypical lymphoid cell infiltration with an angiocentric pattern and extensive necrosis by immuno-histochemical analysis, these cells were positive for $\mathrm{CD} 4, \mathrm{CD} 56$, granzyme $\mathrm{B}$ and negative for $\mathrm{CD} 8, \mathrm{CD} 123, \mathrm{CD} 20$ and $\mathrm{CD} 30$. T-cell rearrangement and Epstein-Barr-virus (EBV) in situ hybridation studies were negative. The patient underwent 5 cycles chemotherapy SMILE regimen monthly sandwiched with radiotherapy on the residual lesions of the right leg with great tolerance but he relapsed within 8months with skin, blood, bone marrow, lung, and cerebrospinal involvement. Based on these findings, the patient was diagnosed with $\mathrm{CD} 4+/ \mathrm{CD} 56+$ hematodermic neoplasm (blastic NK-like T-cell lymphoma) treated with one course of hyper-CVAD regimen, he died within 20 days with a septic chok. Despite the use of L-Asparaginase and radiotherapy the prognosis is very poor; we suggest the exploration for highly active drugs, hematopoietic stem cell transplantation (HSCT) is crucial to improve survival.
\end{abstract}

Keywords: Blastic NK-Like T-cell Lymphoma; Hyper-CVAD; SMILE; Prognosis; Treatment

\section{Introduction}

Since the early 1990s, several cases of CD4+ CD56+ hematodermic neoplasm's (HN) (WHO-EORTC) have been reported. Based on the lymphoid morphology of tumor cells, expression of CD56 (also known as neural cell adhesion molecule NCAM) and the absence of the T-cell receptor or surface CD3, a natural killer (NK) origin has been suggested, despite the negativity of most NK-associated markers and the absence of azurophilic granulations. These tumors fell under the designation of blastic NK-like T-cell lymphoma (WHO) without an evidence of a NK-cell origin [1]. From 1993 to 2011, 64 cases of cutaneous lymphoma were diagnosed in our hospital, with only one case of blastic NK-like T-cell lymphoma of the skin in 2011. Due to the rarity of this entity, we report a case of a 49-year old man with blastic
NK-like cell lymphoma with skin, bone marrow, blood, cerebrospinal fluid involvement and reviewed clinical manifestations, treatment and prognosis of this condition.

\section{Case Report}

A 49-year-old Tunisian man developed slowly growing subcutaneous $15 \mathrm{~cm}$ nodules secondly ulcerated localized in the right leg. Subsequently similar nodules appeared on the trunk, back, the other leg and upper extremities. At the time of evaluation, about 10 red nodules from index finger seized to thumb seized and homolateral inguinal lymphadenopathy were noted (Figures 1(A)-(C)).

Skin biopsy showed atypical lymphoid cell infiltration with an angiocentric pattern and extencive necrosis. These cells were positive for CD4, CD56, CD123 granzyme B, and negative for $\mathrm{CD} 8$, terminal deoxynucleotidyl trans- 

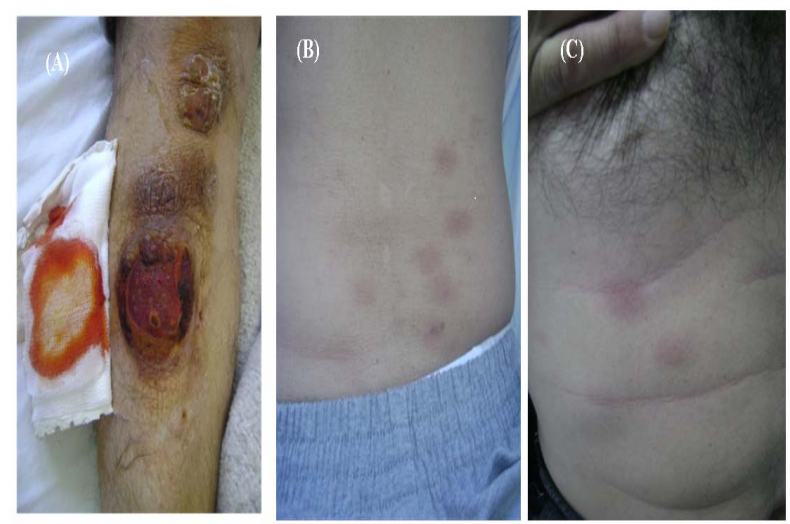

Figure 1. Skin lesions before treatment. (A) Ulcerated nodules with a fibrinous base of the right leg before chemotherapy; (B) Violaceous skin nodules on the back before chemotherapy; (C) Skin nodules on the trunk before chemotherapy.

ferase (TdT), CD20 and CD30. T-cell rearrangement and Epstein-Barr-virus (EBV) in situ hybridation studies were negative (Figure 2).

Thus the diagnosis of CD4+ CD56+ hematodermic neoplasm was established. Laboratory evaluation revealed normal level of lactate deshydrogenase (LDH $=332$ $\mathrm{UI} / \mathrm{L}$ ); serum titers for EBV antibodies were negative for IgM and positive for IgG; completed tomographic (CT) scan showed an infracentimetric mediastinal lymphadenopathy. Bone narrow biopsies as well as complete ORL examination were normal. His clinical stage was determined as T3bN1M0 according to the TNM classification. The decision was to treat the patient with 6 courses of SMILE therapy sandwiched with radiotherapy. The patient underwent 3 cycles chemotherapy SMILE regimen monthly based on: Dexamethasone (40 mg, day $2-4$ ), Etoposide $\left(100 \mathrm{mg} / \mathrm{m}^{2}\right.$, day $\left.2-4\right)$, Methotrexate $\left(2 \mathrm{~g} / \mathrm{m}^{2}\right.$ day 1), Ifosfamide $\left(1.5 \mathrm{~g} / \mathrm{m}^{2}\right.$, day $\left.2-4\right)$, L-asparginase $\left(6000 \mathrm{UI} / \mathrm{m}^{2}\right.$, day $8,10,12,14,16,18$ and 20$)$ with radiotherapy on residual lesion after 2 cycles. At the end of the first 3 cycles of chemotherapy only 2 smalls violet papules measuring $6 \mathrm{~cm}$ and $3 \mathrm{~cm}$ respectively in the right leg remained (Figure 3).

Four weeks later he received external radiotherapy at the dose of 45 Gy through direct field on the residual lesions with great tolerance, than he received the other cycles of SMILE chemotherapy. Physical examination revealed no evidence of disease with a follow up of 8 months. While the patient received the fifth cycle of SMILE therapy he presented with erythematous nodules on the trunk and chronic cough, a second skin biopsy demonstrated relapsed dermal lymphoid infiltration. Whole body computed tomography (CT) scan found 2 lung nodules on the median right lobe and 3 left posterior lung

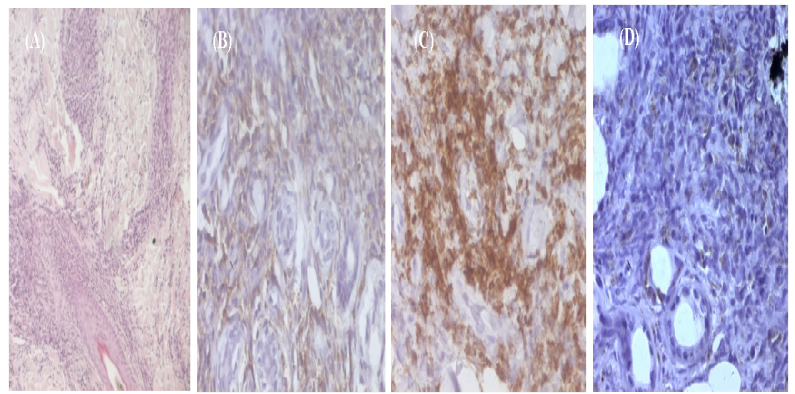

Figure 2. Morphologic and immunohistochemical analysis of the blastic-NK cell lymphoma of the skin. (A) Dense lymphoid infiltrate diffusely involving dermis (Hematoxylin-eosin stain; original 50× magnification); (B) Lymphoid cells staining strongly positive with CD56 marker (original 400× magnification; (C) Lymphoid cells staining strongly positive with CD4 marker (original stain 400× magnification); (D) Lymphoid cells staining strongly positive with granzyme marker (original stain $400 \times$ magnification).

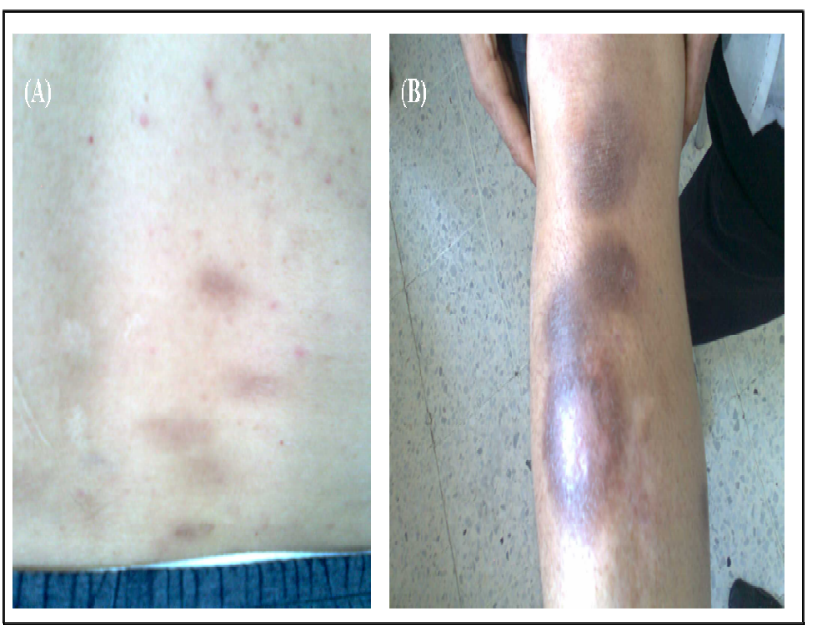

Figure 3. Skin lesions after treatment. (A) Skin lesions of the back after 3 cycles of chemotherapy; (B) Skin lesions of the right leg after chemoradiotherapy.

nodules, bone marrow biopsy was normal. Laboratory investigations, including complete blood count, liver enzymes, serum LDH were within normal limits. Two weeks after achieving the fifth course of chemotherapy, the patient developed weakness, fatigue, fever and dyspnea. Laboratory data was notable for leukocytosis (WBC count $41.9 \times 10^{9} / \mathrm{L}$, with $33 \%$ transformed, apparently lymphoid cells medium sized, basophilic cytoplasm irregular nuclei) (Figure 4(A)), anemia (hemoglobin 8.8 $\mathrm{g} / \mathrm{dl}$ ), thrombocytopenia (platelet count $93 \times 10^{9} / \mathrm{L}$ ), an increase serum LDH level of $1667 \mathrm{UI} / \mathrm{L}$. Circulating malignant cells in the peripheral blood displayed the following phenotype by flow cytometry: CD45+, CD38+, CD56+, CD7+, surface CD3-, cytoplasmic CD3+, CD4-, CD8-, CD57-, CD16-, cMPO-, CD13-, CD33- TCR gama/delta. Bone marrow aspiration showed infiltration 
with $10 \%$ centroblastoid cells with irregular nuclei and inconspicuous nucleoli (Figure 4(B)). Bone marrow biopsy showed massive infiltration with medium-sized blasts (Figure 4(C)).

Examination of cerebrospinal fluid showed infiltration with lymphoid blastic cells. Cytogenetic analysis revealed complex karyotype with 46, XY, del (11)(p14), del (12)(p13) [4]/45, XY, add (1)(p36), del (12)(p13), -22 [3]/45, XY, add (2)(p24), del (12)(p13), -15 [2]/46, $\mathrm{XY}$ [11]. Based on these findings, the patient received the first course of hyper-CVAD regimen (fractionated cyclopho-sphamide, vincristine, doxorubicin, and dexamethasone), with central nervous system prophylaxis using intrathecal methotrexate/cytarabine, he died within 20 days with a septic chok.

\section{Discussion}

Natural killer (NK) and NK-like T-cell lymphoma are aggressive hematologic malignancies that have an extranodal presentation. The main affected organ sites include the gastrointestinal tract, skin and nasal cavities. These neoplasms are categorized into nasal versus non nasal type; they can present initially in the skin and/or involve the skin as a part of a multiorgan disseminated lymphoma. In both NK and NK-like T-cell lymphomas, the neoplastic cells express CD2 and CD56. The distinction of NK versus NK-like T-cell lymphoma is based on the T-cell receptor (TCR) $\beta$ and/or $\gamma$ gene rearrangement and surface $\mathrm{CD} 3$ expression; those that lack these features are categorized by NK lymphomas, those that manifest surface $\mathrm{CD} 3$ and exhibit a TCR rearrangement are considered NK-like T-cell lymphomas. The expression of CD4 is designed as blastic NK-like T-cell lymphoma. More recently, it has been established that the

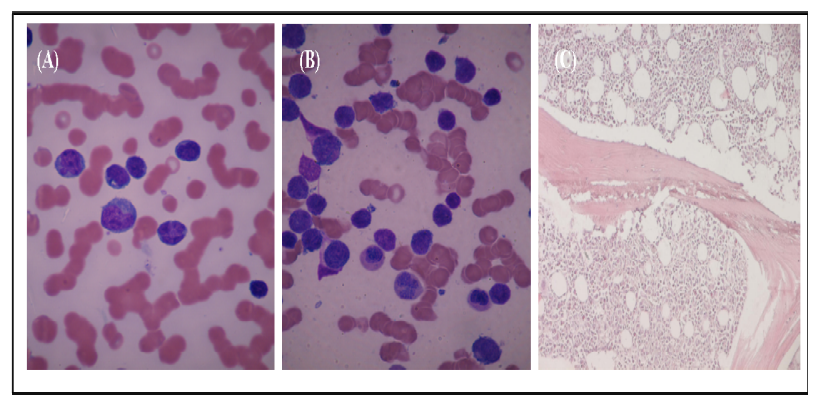

Figure 4. Morphologic analysis of the blastic NK-cell lymphoma in the blood and bone marrow; (A) Lymphoid medium-sized cells, basophilic cytoplasm, irregular nuclei (Blood) (May-Grunwald-Giemsa staining); (B) Bone marrow aspiration: centroblastoid cells with irregulae nuclei, in-conspicuous nucleoi (May-Grunwald-Giemsa staining); (C) Medium-sized blasts infiltrating the bone marrow biopsy (hematoxylin eosin, $\times 100$ ). cell of origin is a plasmacytoid/dendritic cells, conesquently the term "blastic NK-like T-cell lymphomas has been supplanted by the term "CD4+ CD56+ hematodermic neoplasm" in the WHO/EORTC classification but is now termed blastic plasmacytoid dendritic cell neoplasm(BPDCN) [2,3]. Blastic NK-like T-cell lymphoma can arise at any age including childhood, but it tends to present in middle aged or elderly patients with a median age of 52 years [3-5]. It affects predominantly man than woman with a sex ratio 3:1 [5], there is no racial predilection [6]. Similar to acute leukemia, blastic NK-cell leukemia/lymphoma has a multiorgan involvement, lymphadenopathy, splenomegaly with a rapid blood and bone marrow involvement [7]. Cutaneous lesions may be solitary or multiple nodules or tumors, or have petechial appearance witch tend to be generalized [3,7-9]. Skin biopsy reveals a dense dermal infiltration by medium malignant cells without epidermal involvement with focal angiocentric accentuation and necrosis $[2,4,7]$. Immunophenotypically, blastic NK-cell lymphoma is typically $\mathrm{CD} 45+, \mathrm{CD} 2+$, surface $\mathrm{CD} 3-$, cytoplasmic $\mathrm{CD} 3+$, $\mathrm{CD} 4+, \mathrm{CD} 56+, \mathrm{CD} 5-, \mathrm{CD} 7+/-, \mathrm{TdT}+/-\mathrm{TCR}$ genes are in germline configuration [2,7]. The absence of positivity for myeloperoxidase, $\mathrm{CD} 13$ and $\mathrm{CD} 33$ in bone marrow cells excluded the diagnosis of CD56+ myeloid leukemia $[4,10]$. In contrast with aggressive NK-cell leukemia and extranodal NK/T-cell lymphomas, EBV DNA in tumor cells is negative, so the possibility of extranodal NK/ T-cell lymphoma nasal type is excluded and the positivity of EBV might serve as a key point for differential diagnosis from mature NK cell malignancies $[8,9]$. $\mathrm{Cy}-$ togenetic analysis reveals a complex karyotype, such as deletion of chromosomes $9,13,15$, deletion of the $5 \mathrm{q}$, $12 p$ and $6 q$ abnormalities, but no recurring abnormalities were identified [7]. Deletion of $12 p$ and add 1 were found in our patient. Suzuki et al. reported 4 cases of blastic NK cell lymphoma, only one patient had cytogenetic abnormalities [10]. Differential diagnosis includes other CD56+ lymphomas with cutaneous involvement. These include extranodal NK/T-cell lymphoma (ENKTL) nasal type, CD56+ myeloid leukemia, cutaneous CD30+ lymphoproliferation with CD56 expression and cutaneous granulocytic sarcoma $[2,8]$. The prognosis of patients with blastic NK-like T-cell lymphoma is poor with a median survival of 14 months, 2 and 5 years overall survival of $33 \%$ and $6 \%$ respectively $[7,8]$. There is no standard treatment for this malignancy, response to combination chemotherapy such as CHOP, is minimal or transient and early progression is common. Radiotherapy is effective for localized disease only [8]. More aggressive therapies directed to allogenic stem cell transplantation are probably superior with long term remission [8]. Several studies showed efficacy of regimens containing L-Asparaginase. 
The rationale is based on reports that L-Asparaginase is effective in the treatment of NK/T-cell lymphoma $[8,11]$. L-Asparaginase induced selective apoptosis of NK-lymphoma cells in vitro, these results in fast inhibition of DNA and RNA synthesize in lymphocytes [5]. Etoposide has demonstrated in vitro and in vivo efficacy. Increased expression of a multidrug resistant (MDR) phenotype has been correlated with aggressive behavior in these lymphomas $[3,5,6,10]$. MDR phenotype and P53 mutation also can be associated with a poor prognosis and may help predict response to the treatment. $[3,5,6,10]$. The components of SMILE protocol are MDR unrelated agents and EBV associated lymphoproliferative disorders. Motoko et al. reported an overall response rate at $67 \%$ for six Japanese patients with advanced-stage, relapsed or refractory ENKT-CL and leukemia treated with six courses of chemotherapy SMILE regimen with sandwiched radiotherapy after three courses [12]. HyperCVAD regimen is used mainly for therapy of acute lymphocyte leukemia and high-grade lymphomas. Shapiro et al. reported successfully achieved complete remissions in two patients using the hyper-CVAD regimen [7]. Among five patients with $\mathrm{CD} 4+/ \mathrm{CD} 56+\mathrm{HN}$, one patient treated with the hyper-CVAD regimen achieved a complete response and remain alive with a follow-up of over than 38 months [13]. Allogenic HSCT, with the potential benefit of graft-versus lymphoma effect is a second option for patients with advanced disease, long remission of blastic NK-cell lymphoma was reported after autologous peripheral blood stem cell transplantation. Therefore allogenic or autologous, or cord blood stem cell transplanttation is a promising treatment strategy, but small series have shown that it is a potentially curative option $[7,8$, $14,15]$. Age $\leq 40$ years, presentation with only skin lesions, initial treatment with transplantation directed regimens and TdT expression by $>50 \%$ of the neoplastic cells were associated with better prognosis [2].

\section{Conclusion}

CD56+ CD4+ HN's are an extremely difficult group, for pathologists and clinicians. For pathologists, correct classification of these lymphomas is difficult, expensive and time-consuming, it requires application of several complimentary techniques such as extensive phenotyping, EBV analysis and T-cell receptor TCR gene rearrangement studies. Clinicians are confronted with an aggressive clinical behavior and a fatal outcome often within 1 year after diagnosis, and may consider more intensive therapies, as in acute leukemias, as initial therapy. Prospective data on larger series of patients treated homogeneously also with innovative approaches are needed in order to establish the best treatment for this disease.

\section{REFERENCES}

[1] R. Dummer, K. Asagoe, A. Cozzio, G. Burg, U. Doebbeling and P. Golling, "Recent Advances in Cutaneous Lymphomas," Journal of Dermatological Science, Vol. 48, No. 3, 2007, pp. 157-167. doi:10.1016/j.jdermsci.2007.09.001

[2] C. M. Magro, P. Porcu, J. S. Schaefer, J. W. Erter, R. R. Furman, P. K. Shitabata, et al., "Cutaneous CD4+ CD56+ Hematologic Malignancies," Journal of the American Academy Dermatology, Vol. 63, No. 2, 2010, pp. 292-308. doi:10.1016/i.jaad.2009.08.044

[3] R. G. Asher and K. Hollowood, "Primary Cutaneous Lymphoma: An Overview Based on the WHO-EORTC Classification, Mini-Symposium: Haematopathology Update III," Diagnostic Histopathology, Vol. 16, No. 4, 2010, pp. 168-181.

[4] K. K. Sra, J. W. Labiche, R. Rapini, R. Jordon, S. Raimer and S. Tyring, "T/Natural Killer-Cell Lymphomas," Journal of the American Academy Dermatology Letters, 2005, pp. 708-709.

[5] M. W. Bekkenk, P. M. Jansen, C. J. L. M. Meijer and R. Willemze, "CD56+ Hematological Neoplasms Presenting in the Skin: A Retrospective Analysis of 23 New Cases and 130 Cases from the Literature," Annals of Oncology, Vol. 15, No. 7, 2004, pp. 1097-1108.

doi:10.1093/annonc/mdh268

[6] Y. L. Kwong, "Natural Killer-Cell Malignancies: Diagnosis and Treatment," Leukemia, Vol. 19, No. 12, 2005, pp. 2186-2194. doi:10.1038/sj.leu.2403955

[7] M. Shapiro, M. A. Wasik, J. M. Junkins-Hopkins, A. H. Rook, C. C. Vittorio, H. Ikatura, et al., "Complete Remission in Advanced Blastic NK-Cell Lymphoma/Leukemia in Elderly Patients Using the Hyper-CVAD Regimen," American Journal of Hematology, Vol. 74, No. 1, 2003, pp. 46-51. doi:10.1002/ajh.10381

[8] J. W. Tjiu and C. H. Hsiao, "Blastic Natural Killer-Cell Lymphoma Presenting in the Skin," Tzu Chi Medical Journal, Vol. 19, No. 3, 2007, pp. 173-178.

http://health.elsevier.com/tcmj

[9] R. P. Falcao, A. B. Garcia, M. G. Marques, B. P. Simoes, B. A. Fonseca and M. L. Rodrigues, et al., "Blastic CD4 NK Cell Leukemia/Lymphoma: A Distinct Clinical Entity," Leukemia Research, Vol. 26, No. 9, 2002, pp. 803-807.

[10] R. Suzuki and S. Nakamura, "Malignancies of Natural Killer (NK) Cell Precursor: Myeloid/NK Cell Precursor Acute Leukemia and Blastic NK Cell Lymphoma/ Leukemia," Leukemia Research, Vol. 23, No. 7, 1999, pp. 615-624.

[11] J. V. E. Reyes, T. Al-Saleem, V. G. Robu and M. R. Smith, "Extranodal NK/T-cell Lymphoma Nasal Type: Efficacy of Pegaspargase. Report of Two Patients from the United States and Review of Literature," Leukemia Research, Vol. 34, No. 1, 2010, pp. 50-54. doi:10.1016/j.leukres.2009.09.002

[12] M. Yamaguchi, R. Suzuki, Y. L. Kwong, W. S. Kim, Y. Hasegawa, J. I. Kiya, et al., "Phase I Study of Dexamethasone, Methotrexate, Ifosfamide, L-Asparaginase 
and Etoposide (SMILE) Chemotherapy for AdvancedStage, Relapsed or Refractory Extranodal Natural Killer (NK)/T-cell Lymphoma and Leukemia," Cancer Science, Vol. 99, No. 5, 2008, pp. 1016-1020. doi:10.1111/j.1349-7006.2008.00768.x

[13] P. Ng Ashley, L. Stephen, R. Timothy, Mc. Cormack, C. Prince and H. M. D. Awesterman, "Primary Cutaneous CD4+/CD56+ Hematodermic Neoplasm (Blastic NK-Cell Lymphoma): A Report of Five Cases," Hematologica, Vol. 91, No. 1, 2008, pp. 143-144.

http://www.haematologica.org/journal/2006/01/143html
[14] J. J. Leitenberger, C. N. Berthelot, K. D. Polder, P. McLaughlin, D. Jones and M. Duvic, "CD4+ CD56+ Hematodermic/Plasmacytoid Dendritic Cell Tumor with Response to Pralatrexate," Journal of the American Academy Dermatology, Vol. 58, No. 3, 2008, pp. 480-484. doi:10.1016./jaad.2004

[15] E. C. Parlette, Z. Elliott, F. W. Hall and B. S. Grabam, "Primary Cutaneous Blastic Natural Killer Cell Lymphoma," Journal of the American Academy Dermatology, Vol. 53, No. 4, 2005, pp. 742-743. 